\title{
In situ Methane Hydrate Morphology \\ Investigation: Natural Gas Hydrate-bearing \\ Sediment Recovered from the Eastern Nankai \\ Trough Area
}

Yusuke Jin, ${ }^{1}$ Yoshihiro Konno, ${ }^{1}$ Jun Yoneda,${ }^{2}$ Masato Kida, ${ }^{1}$ and Jiro Nagao ${ }^{1}$

\footnotetext{
${ }^{1}$ Methane Hydrate Production Technology Research Group, Research Institute of Energy Frontier, Department of Energy and Environment, National Institute of Advanced Industrial Science and Technology (AIST), Tsukisamu-Higashi, Toyohira-Ku, Sapporo 062-8517, Japan

${ }^{2}$ Methane Hydrate Geo-mechanics Research Group, Research Institute of Energy Frontier, Department of Energy and Environment, The National Institute of Advanced Industrial Science and Technology (AIST), 16-1 Onogawa, Tsukuba, Ibaraki, 305-8569, Japan
} 


\section{Grain-size distribution}

Grain-size distribution of sample was measured using a laser-diffraction particle size analyzer (Microtrac MT3300 EX, Nikkiso Co. Ltd., Japan). Details of measurement procedure are described in literature. Figure S1 show Grain-size distribution of AT1-C-10P sample that is close depth to AT1-C-10P (286.65-286.77 mbsf).

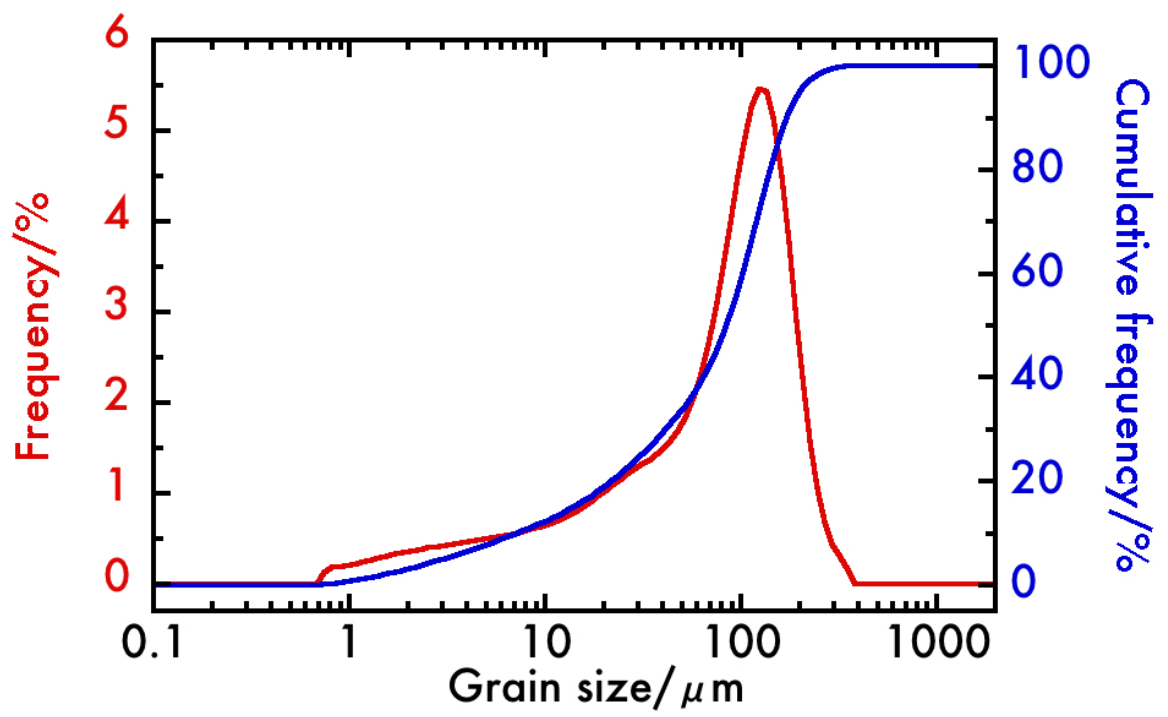

Figure S1 Grain-size distribution of AT1-C-10P. Red and blue lines indicate frequency and cumulative frequency of grains.

\section{PNATs-X}

Figure S2 show the schematic of PNATs-X. Resolution range in an obtained CT image via our PNATs-X can be varied from ca. $29 \mu \mathrm{m} /$ pixel (XY-plane of the field of view: 30 $\mathrm{mm}$ ) to ca. $127 \mu \mathrm{m} /$ pixel ( $X Y$-plane of the field of view: $130 \mathrm{~mm}$ ) by geometric 
configuration of an object. Whole X-ray CT images (maximum core length:120 cm) can be measured by moving height of X-ray source and FPD.

X-ray shielding box

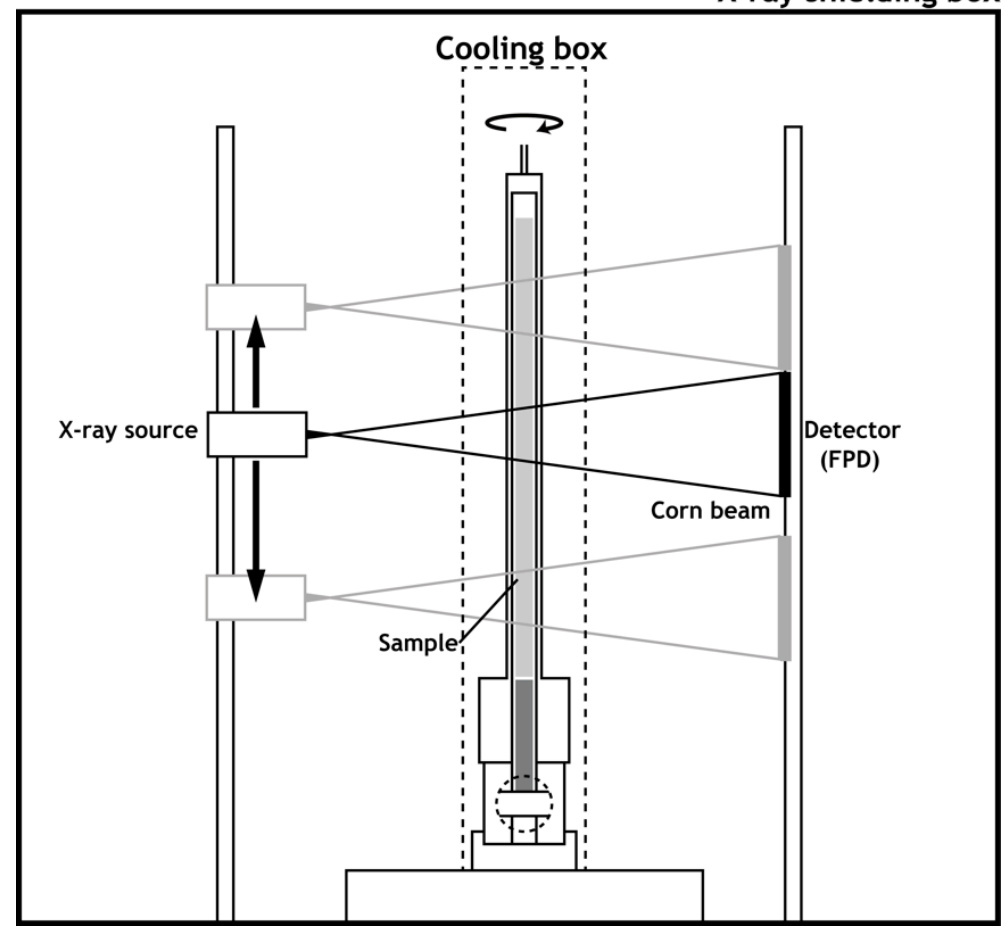

Figure S2. The schematic of PNATs-X (X-ray CT system)

\section{PWV profiles via PNATs-AIST-IPTC}

Figure S3 show the schematic of PWV measurement by a combination of PNATsAIST-IPTC and PWV probe. P-wave oscillator and receiver directly contact to core surface through holes in a liner. 


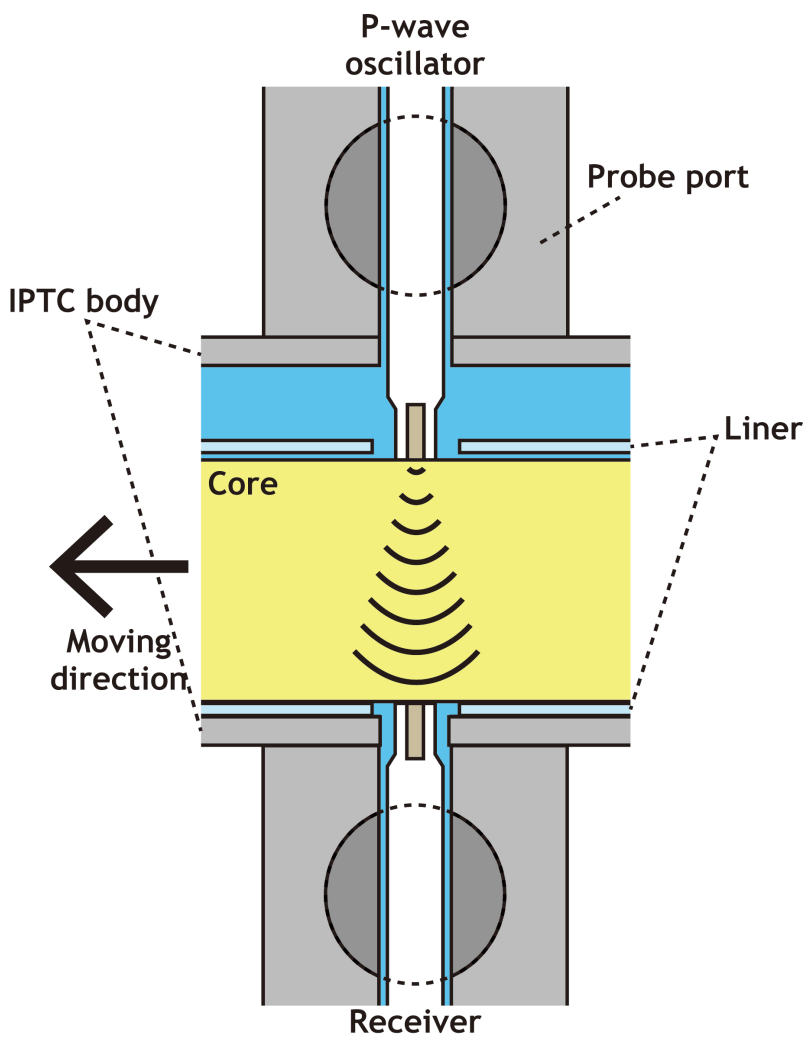

Figure S3. The schematic of PWV measurement through the PNATs-AISTIPTC and PWV probe.

\section{Density profiles}

Density of sediment sample was measured using a gamma-ray density meter (PNATs-PG-Gamma: PM-1000, Nanogray Inc., Japan). Density values of AT1-C core samples were evaluated from PNATs-PG vessel (Stainless steel, thickness $=15 \mathrm{~mm}$ ), plastic liner (polycarbonate, $3 \mathrm{~mm}$ in thickness), and sample (thickness $=$ ca. $50 \sim 53.7$ $\mathrm{mm}$ ) between gamma-ray source and detector. Density profiles can be collected every 
$0.5 \sim \mathrm{cm}$. The density value was estimated by time averaging of $120 \mathrm{sec}$. The uncertainty of the density measurement was $\pm 0.0075 \mathrm{~g} / \mathrm{cm}^{3}$. Measurements were performed at 278 $\mathrm{K}$ and $10 \mathrm{MPa}$. The schematic of PNATs- PG-Gamma was described in Figure S4.

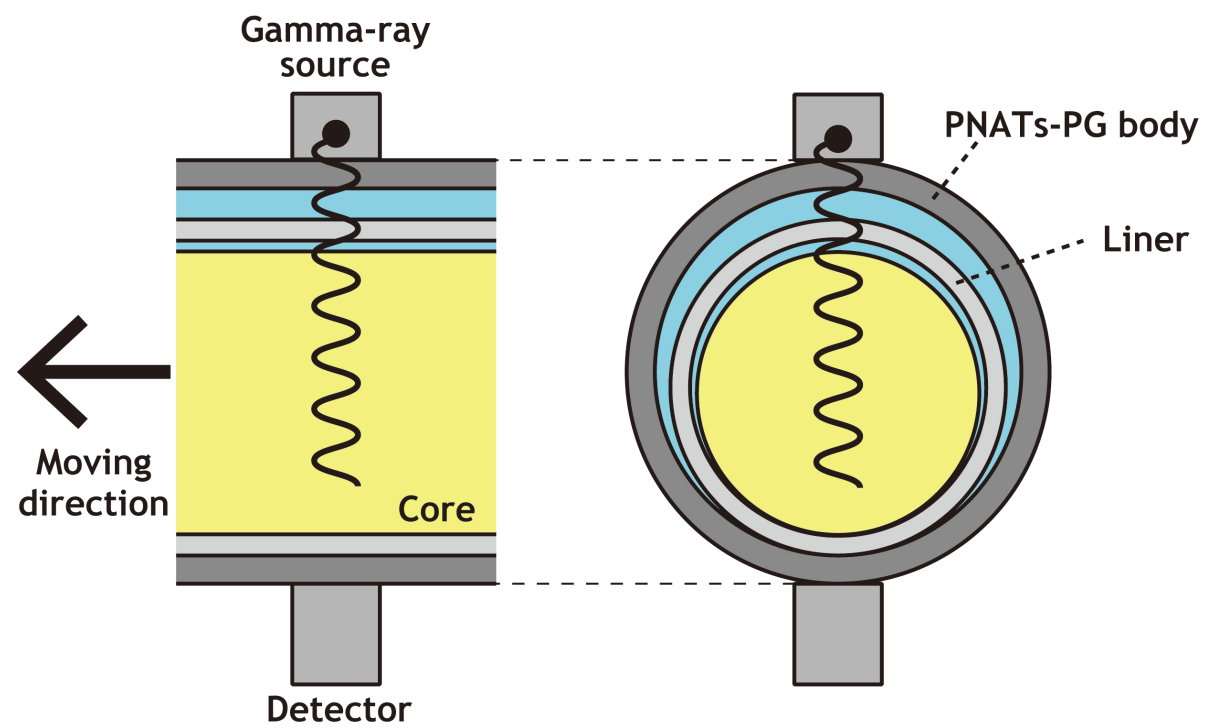

Figure S4. The schematic of PNATs-PG-Gamma (gamma-ray density meter system) 